\title{
A Conversation Between Christoph Zindel and Ken Herrmann
}

\author{
Christoph Zindel ${ }^{1}$ and Ken Herrmann ${ }^{2}$ \\ ${ }^{I}$ Siemens Healthineers, Erlangen, Germany; and ${ }^{2}$ Department of Nuclear Medicine, Universitätsklinikum Essen, Essen, Germany
}

$\mathbf{K}$ en Herrmann, MD, MBA, a professor of nuclear medicine at the Universitätsklinikum Essen (Germany), talked with Christoph Zindel, MD, a member of the Managing Board of Siemens Healthineers (Erlangen, Germany). In his previous role, Dr. Zindel was the president of Diagnostic Imaging at Siemens Healthineers. He received his medical degree from J.W. Goethe University (Frankfurt, Germany) and was a resident in surgery, internal medicine, and nuclear medicine at the University of Tübingen (Germany). He has been with Siemens through most of his career, leading teams in urology and MR before becoming CEO of PETNET, Siemens Healthcare USA.

Dr. Herrmann: Thank you very much for making time for this interview. You are a new board member of Siemens Healthineers and were previously president of Diagnostic Imaging for the company. Could you tell us about your background and career before becoming a board member?

Dr. Zindel: First of all, thank you for inviting me! It is my great pleasure to give an interview for you and this journal. It reminds me of my medical studies as I did my MD thesis in nuclear medicine, developing a large software project in the context of bone scintigraphy. Thus, my background-to come to your question-is medical. I did my residency in surgery and my research focused on endoscopic procedures and therapies. To join the industry was a decision I made in 1998. The decision wasn't easy but I haven't regretted it at all. I started at Siemens Healthcare, which was back then a business area of Siemens and is nowadays operating under the name Siemens Healthineers as a separate listed company. During my first years, I learned a lot about urology (e.g., x-ray equipment and lithotripters). Then, I took the opportunity to join our MR business and within 12 years I worked in nearly every department of our MR business, including research, development, and marketing. This time significantly broadened my horizon. However, after 12 years at MR I decided to move to the United States where I became the CEO of PETNET, our radiopharmaceutical business. This was quite a change and a step out of what we often call the "comfort zone" due to new geography, new culture, new business model, and other challenges. In 2014, I took a tough decision to leave the company to localize in the United States and work for a local company. But this decision lasted only for 1 year because I was asked to re-join Siemens Healthineers to lead the MR business. This was a great honor and I wanted to do this since MR is such an exciting field with so many inspiring people, even if this meant that I had to move from Miami to Erlangen. Afterward, everything happened quickly: In 2018, I was appointed as president of the whole business area Diagnostic Imaging. This was another challenge in my life because of the complexity of this business area. Last fall, the

COPYRIGHT @ 2020 by the Society of Nuclear Medicine and Molecular Imaging. supervisory board of Siemens Healthineers appointed me as a member of the Managing Board, which was certainly an extraordinary privilege.

Dr. Herrmann: Diagnostic imaging plays a big role for Siemens Healthineers. What do you see as the major future trends in diagnostic imaging in general, not only in nuclear medicine?

Dr. Zindel: Future developments in diagnostic imaging will be driven by challenges the health-care market faces: limited or even no access to medical

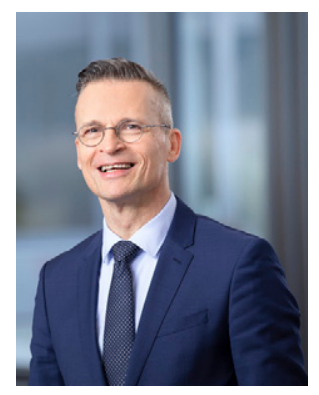

Christoph Zindel, MD care for $50 \%$ of the world's population, an estimated shortage of around 15 million health-care workers by 2030, and increasing demand for more sophisticated medical services in emerging countries. These are only a few examples of challenges. I would like to highlight 3 approaches to meet these challenges:

Number 1 is simplification. To address the shortage of health-care workers and to make diagnostic imaging available in underserved areas or diagnostic networks, it is important to simplify current modalities. We recently introduced at the Radiologic Society of North America meeting a new push-button CT that is very easy to operate. We do the same for MR. And we presented a mobile CT that can be brought easily to patients. Simplification sounds easy, but it is not. We had to invest intensively in expertise to simplify and optimize the modalities. If you want to move complexity from the front end to the backbone, you need a lot of innovative minds.

Number 2 is remote operation. This is a process that started in Brazil with a highly consolidated imaging market in which some entities operated up to $100 \mathrm{MRs}$ and CTs and needed to streamline protocols and centrally operate the devices. We developed a remote-control system that allows device operation over long distances, bringing expertise into areas where that expertise does not exist. Locally, a nurse is available onsite for patient safety. The obvious benefit is the possibility of addressing the problem of fewer health-care workers while ensuring high standards of care.

Number 3, the future is about digitalization and artificial intelligence (AI). But we do not drive this for the sake of digitalization, but rather to support clinical decision making, ensuring quality and productivity. Decades back, my MD thesis was about clinical decision support to semiautomate interpretation and documentation in bone scintigraphy. Back then, we discussed many expert systems, but none of them was realized, because of the lack of technology and reluctance to accept so-called expert systems.

Today, we can clinically implement decision support systems. Our AI-Rad Companion imaging decision support system, which recently received U.S. Food and Drug Administration approval, automatically analyzes CT chest data and even more complex MR 
data. We specifically named it "companion," because we believe that these systems will not replace imaging professionals but, instead, free them up and help them to ensure quality through clinical decision making by AI.

Dr. Herrmann: The big challenge is that you are expected to provide basic, simple imaging to the $50 \%$ of the world who are completely underserved, as well as being the lead innovator to address the expectations of the other $50 \%$ who are constantly searching for the next new thing. How do you address these needs as a company?

Dr. Zindel: You are right with your observation-we are facing different markets. The emerging markets with a population deserving and increasingly demanding medical services, and the consolidated markets that request tools for efficient handling of scanner fleets, etc. But facing these two markets is an exciting opportunity. We use innovations from the research segment and transfer them into cost-efficient products. In addition, we change the way in which care is delivered by remote access strategies, AIassisted, and robots. The real challenge is to move AI-based tools through the regulatory processes. We need to make sure that the tools work adequately and pass regulatory scrutiny, which is quite a new field and a challenge.

Dr. Herrmann: What are other areas where, in your view, AI is going to significantly impact imaging?

Dr. Zindel: Let me compare us with the automotive industry's move toward autonomous driving. Their lowest tier is defined as patient, with all the same functions that can be simulated (muscles, contractility, function, conductive system, etc.). We do that to identify patients who will most likely respond to an electrophysiologic intervention to treat their atrial fibrillation. Such an intervention could cost around $€ 20,000 € € 25,000$, depending on the country's health system. Today many patients do not respond at all to such an intervention, so identifying the ones in whom it is most likely to succeed is very important to the patient as well as economically.

Dr. Herrmann: This sounds like creating an avatar allowing simulation of whether an intervention will be successful or not.

Dr. Zindel: Correct. And we are already going beyond the heart. We have now started modeling the liver, which is a challenging but fascinating organ. The final goal is to model the whole human body - this is many years out but is the ultimate goal. At each development stage, however, it is possible to derive information for certain diseases and certain therapeutic approaches.

Dr. Herrmann: Nowadays diagnostics encompass more than just imaging, and it appears appealing to integrate imaging with other diagnostic disciplines, such as laboratory medicine and pathology. Some newly created departments unite all these disciplines in superdiagnostics or integrated diagnostics units. What is your vision there?

Dr. Zindel: There is indeed such a trend, and we see it among our customers. There is a tendency to bring, for example, imaging and pathology together as integrated diagnostics. From a higher level it makes perfect sense to combine in vitro and in vivo data.

\section{"Future developments in diagnostic imaging will be driven by challenges the health-care market faces: limited or even no access to medical care for $50 \%$ of the world's population, an estimated shortage of around 15 million health-care workers by 2030, and increasing demand for more sophisticated medical services in emerging countries."}

assistance, such as a lane assist. The next levels include automatic driving with some driver input, but driver awareness is still necessary. The highest level (and the ultimate goal) is the completely autonomous car with no input needed from the driver.

This analogy can be translated to our imaging business. The lowest tier and the equivalent of things like lane assistants is automatic positioning before a CT scan, image reconstruction, reduction of noise, and so on, which are all support systems.

Our equivalents to autonomous driving with some input and awareness are already on the market as well. Our AI-Rad Companion looks at images as a kind of a second reader and highlights areas at which the radiologist should look more closely. The radiologist as the "driver" is in full control, but AI helps with standardized and often tedious tasks, such as measuring the diameter of an aorta or counting lesions in a mammogram. They help by augmenting the radiologist's abilities; highlighting abnormalities he or she might have missed or that were simply not the area of focus.

Our equivalent to fully autonomous cars on the highest level as defined by the car industry is our vision of the "digital twin." Here we diverge somewhat from the analogy, because the physician/radiologist will always be the one in charge and all digital systems will support decision making but will probably never replace them. The aim of the digital twin is to digitally mirror human physiology. We have already developed a digital model of the heart, based on imaging and electrocardiographic data. It is a digital model of the real heart of the
Additional data are available for integration (e.g., genetic information), and this more comprehensive data can be used to arrive at a more precise and more individualized therapeutic concept.

We recognized this trend years ago and have started the development of the product called AI-Pathway Companion. It is again a companion, as we don't intend to replace doctors but to support them. The current prototype focuses on prostate cancer and combines imaging data, in vitro data, and even longitudinal prostatespecific antigen data and then compares the individual patient to medical standards. This system includes the most important guidelines and is automatically updated when the guidelines change. The AI-Pathway Companion is designed to provide data-driven therapeutic options. In areas with limited expertise, this could also ensure quality when addressing malignant, life-threatening diseases.

Dr. Herrmann: You mentioned prostate cancer, and obviously the prostate-specific membrane antigen tracer family provides a lot of new information. Do you think that PET tracers providing information about receptor status will see significant future growth?

Dr. Zindel: To answer from a higher level without hurting regulatory and reimbursement discussions for PET tracers, there is no doubt that PET has a bright future ahead. That is why we invest in digital PET/CT, PET/MR systems, and other exciting new developments aiming at higher resolution and speed. Obviously, the combination of PET with theranostics as companion 
biomarkers is very exciting as well, so PET usage has definitely not reached its ceiling. PET is a very vibrant and exciting business field.

Dr. Herrmann: You mentioned theranostics, which are really extending and even shifting the focus of nuclear medicine more toward therapy. How does this impact your company, which is more oriented toward diagnosis than therapy?

Dr. Zindel: Our company has a core in diagnostic imaging but also in advanced therapies, and we are expanding intensively into adjacent fields. Looking back, we integrated in vitro in the last decade with significant investments. Now we are expanding into digital and, of course, are also eyeing the advanced therapeutics space. As an example, we recently acquired Corindus, which specializes in robotic therapy for minimally invasive procedures. We intend to perform these minimally invasive vascular procedures remotely in the heart, in the brain, and other vessels. Indeed, interventional radiology services can be offered in areas in which local expertise is not available. And this is not the end of the story. There is huge potential. When you think about collaborations between academia and industry, we can provide infrastructure, academia can provide excellent expertise, and jointly we can provide quality of care.

Dr. Herrmann: When I see how inefficiently many academic hospitals are run and how efficiently industry is run, why does a company like Siemens Healthineers (or GE or Philips or any other company) not go for complete vertical integration, building and operating hospitals and potentially providing turnkey solutions, especially in underdeveloped countries?

Dr. Zindel: Vertical integration can make sense. Let's say vertical integration involves taking an imaging device such as MR and adding MR-compatible catheters-then it would be beneficial to also have the biopsy and specimen process. If these all come from one vendor, data consistency and workflow efficiency would strongly benefit. We are considering this and also discussing end-to-end workflows.

Moreover, as previously mentioned, there is a lot of consolidation ongoing in the Americas and even in China, resulting in bigger and bigger customers. With this high consolidation rate, it is conceivable that we could do part of the operation, and, to a certain degree, we are already doing this. We can provide the equipment, the maintenance for that equipment, and even personnel support, but the hospital has to bring in the medical doctors.
Dr. Herrmann: If they have doctors, they are happy to bring them in. If they don't, however (and you mentioned the projected deficit of 15 million health-care workers in 2030), they might be happy for you to bring the doctors in or at least provide remote reporting and remote treatment. Isn't this a huge business opportunity?

Dr. Zindel: You are spot on. We could help with remote support, and we could even help with networks of medical doctors where the legal frameworks and regulations allow. We are open to such business models. However, we definitively don't want to intrude into our customers' areas of expertise by operating hospitals or even owning hospitals.

Dr. Herrmann: Siemens Healthineers is quite a big company. When we talk about software development, digital development, sometimes big companies move slower than small, lean startups. What is your approach in this very innovative field? When do you opt for outsourcing versus clean in-house development?

Dr. Zindel: What you are addressing is the field of open innovations. We know very well what our core competencies are. We do, of course, monitor fast-moving startups. If we find someone complementary to us, we have different modes of collaborating, ranging from strategic collaboration up to full integration. The era of developing everything at one location has passed. It is really about using an international network of experts and innovators and bringing them together. We have our own entities all around the world. And, of course, we have a very strong footprint among engineers and other experts in Germany. We are very proud to collaborate with more than $90 \%$ of the top 100 academic institutions in the world. To summarize, a company needs to think carefully about what makes sense and how to best help its customers.

Dr. Herrmann: Finally, a personal question. If you had a chance to go back in time, having the experience and knowledge you have today, would you do it all over again? Or, to be more specific, would you still study medicine?

Dr. Zindel: Definitely yes! Studying medicine offers so many possibilities. For me the combination of medicine and technology was always fascinating. As a team member of Siemens Healthineers, I wake up every morning with a passion for the health of humans. That's what my heart beats for. But everyone needs to find that out for themselves.

Dr. Herrmann: Thank you very much for your time and this discussion. 